\title{
Is Dysphagia Under Diagnosed or is Normal Swallowing More Variable than We Think? Reported Swallowing Problems in People Aged 18-65 Years
}

\author{
Paula Leslie $^{1}{ }^{\mathbb{C}} \cdot$ David G. Smithard ${ }^{2,3}$
}

Received: 27 August 2020 / Accepted: 4 November 2020 / Published online: 23 November 2020

(c) The Author(s) 2020

\begin{abstract}
Purpose Dysphagia prevalence in younger community dwelling adults and across nations is sparse. We investigated the prevalence of swallowing problems in an unselected cohort of people aged 18-65 years.

Methods The EAT-10 Assessment Tool was converted into an anonymized online survey. Invitations were e-mailed to author contacts and onwards dispersal encouraged. Analysis was performed using non-parametric test for group comparison (Mann-Whitney $U$ ) and Spearman's rho correlation.

Results From March 2014 to October 2017: 2054 responses (32 reported ages outside of 18-65 or undeclared) from Africa, Asia, Australasia/Oceania, Europe, and North and South America. Responses: 1,648 female, 364 male, (10 reported as both), median age 34, (range 18-65, mean 37.12, SD 12.40) years. Total EAT-10 scores: median 0 (range 0-36, mean 1.57, SD 3.49). EAT-10 score $\geq 3$ (337) median 5 (range 3-36, mean 7.02 SD 5.91). Median age 36 (range 19-65, mean 37.81, SD 13.21) years. Declared sex was not statistically significantly associated with non-pathological vs. pathological EAT-10 score $(p=0.665)$. Female scores (median 0.00 , mean 1.56 , SD 3.338 ) were significantly higher than for males (median 0.00 , mean 1.62, SD 4.161): $U(\mathrm{Nfemale}=1648, \mathrm{Nmale}=364)=275,420.000, z=-2.677, p=0.007$. Age and EAT-10 score were not associated: females $r_{\mathrm{s}}=-0.043, p=0.079, N=1648$, males $r_{\mathrm{s}}=-0.003, p=0.952, N=364$. Considerable impact on people: "I take ages to eat a main course ... This is embarrassing and I often leave food even though I am still hungry." (no diagnosis, EAT-10 $=17$ ).

Conclusion Concerns regarding swallowing exist in people undiagnosed with dysphagia, who may feel uncomfortable seeking professional help. Dysphagia may be under reported resulting in a hidden population. Subtle changes are currently seen as subtle markers of COVID-19. Further work is required to ensure that what is an essentially normal swallow does not become medicalized.
\end{abstract}

Keywords Dysphagia $\cdot$ Community $\cdot$ Young $\cdot$ Adult $\cdot$ Diagnosis

David G. Smithard

david.smithard@nhs.net

$1 \quad$ School of Sports \& Health Sciences, University of Central Lancashire, Preston PR1 2HE, UK

2 Geriatric Medicine, Queen Elizabeth Hospital, Lewisham and Greenwich NHS Trust, Stadium Road, Woolwich, London SE18 4QH, UK

3 University of Greenwich, London, UK

\section{Introduction}

Eating and drinking are fundamental to the physical and mental well-being of humankind. Eating and drinking are the essential components of identity and connections across society [1,2]. Limitations in how we eat and drink have a profound impact on our lives, irrespective of any other physical, psychological, or mental health co-morbidities. Factors affecting eating and drinking are broad ranging and include physical difficulties in managing the food and drink, intolerances, and in the swallow mechanism itself.

Enjoying food and drink requires the ability to swallow safely. Swallowing is a complex multiphase neuro-muscular function, the main purpose of which is to transfer the food 
or drink (hereafter referred as simply a bolus) safely from the mouth to the stomach. Swallowing is a programmed activity with the primary control being in the brainstem. Cortical input modifies how the bolus moves through the oropharyngeal system depending on the bolus characteristics and number [3]. A safe swallow entails co-ordination between what are typically referred to as the various phases of the swallow [4] and the respiratory cycle [5]. Failure for this to happen will result in problems swallowing (dysphagia). Oropharyngeal dysphagia may be a result of problems with neurological control, muscular control, obstruction, or breathing (cardiorespiratory) [6].

Many medical conditions impact the swallow mechanism and hence affect nutrition, hydration, ingestion of medications, as well as the global human aspects associated with eating and drinking. The prevalence of swallowing problems in many medical conditions such as stroke (8-80\%), dementia (up to $100 \%$ ). and Parkinson's disease (up to $81 \%$ ) is well documented, although there is still a concern of underreporting $[7,8]$.

The prevalence of dysphagia in community dwelling older people ( $>65$ years) has been reviewed over the years. Those studies conducted in the community have used questionnaires such as the EAT-10 designed for those with or without swallowing problems [9] or the Sydney Swallow Questionnaire developed for people with dysphagia and often completed by a nurse or other health care provider if the patient cannot answer [10]. In studies of the elderly where the participants live in the community, the definitions of elderly, the community, and dysphagia vary. This makes comparisons across studies fraught with difficulty; thus; further detail, where required, has been added to allow some comparison between studies (see Table 1) [11-18].

A further complication is that many studies are based on the gastroesophageal literature, and thus a report of "dysphagia" may be indicating a reflux issue, e.g., when dysphagia is simply defined as "In the last year how often had you have difficulty swallowing (a feeling that food sticks in your throat?)" [16]. Reflux issues may not be assessed at all and are thus difficult to rule out [17]. Both of these situations may lead to high figures that do not necessarily indicate an oropharyngeal dysphagia.

Aside from disease and/or age-related dysphagia, there may be a good proportion of the population with swallowing problems that are hidden from health care support. We need to be careful to not go looking for a problem that does not exist or medicalizing the ends of the range of what is normal. Of merit to consider is that the swallow is a finely tuned process involving several systems and as such problems may show subtle pre-existing conditions or herald the onset of certain diseases before other signs. In pseudo-bulbar type motor neuron disease or tiny lacunar type strokes, a change in the swallow may be the first or only sign due to the need for fine co-ordination of systems. Indeed in the current COVID-19 pandemic, evidence is emerging that subtle signs such as taste and smell may be affected early in the disease [19-24]. Taste and smell are inherent components of the sensory system involved in preparing the gastro-intestinal tract to receive, transport, and process each bolus [25]. More obvious issues are clear when we consider what happens to the interplay between breathing and swallowing in conditions including corona virus respiratory infections.

Even if the difficulties are as a result of lying at the edges of the bell curve of normality, people experiencing problems merit access to further investigation and support. Dysphagia impacts people's enjoyment of food and drink, their participation in social activities, and the effectiveness of their medications for other conditions. A part of the difficulty with studies in the world of swallowing is that the populations and definitions vary. One study's "dysphagia" might be another's more specific aspiration event, similarly the "older" population focus of one might be over 65 and in another over 85 years of age.

\section{Objective}

What is the number of people experiencing eating and drinking problems, and what do they report? Prevalence studies have historically targeted small populations, by locality or access to care. There are no large-scale studies looking at the prevalence of reported swallowing problems across nationalities and continents in the age group 18-65 years. We used the Eating Assessment Tool (EAT-10) to investigate the prevalence of dysphagia in multiple countries and across multiple nationalities. This relatively simple tool is one of the most commonly used in larger sample studies and at the time of the study initiation had the most supporting literature [9].

\section{Methods}

\section{Participants}

Participation was open to anyone who received the survey and was aged 18-65 years. The survey was distributed through contacts of the authors, via professional lists, student participation (encouraging parents and other family members), and at professional meetings. Wide onward distribution was encouraged. We have no way of knowing the final pool who received the survey, but only the number of people who completed it. No personally identifying information was collected. All data are shared as participants reported them. 


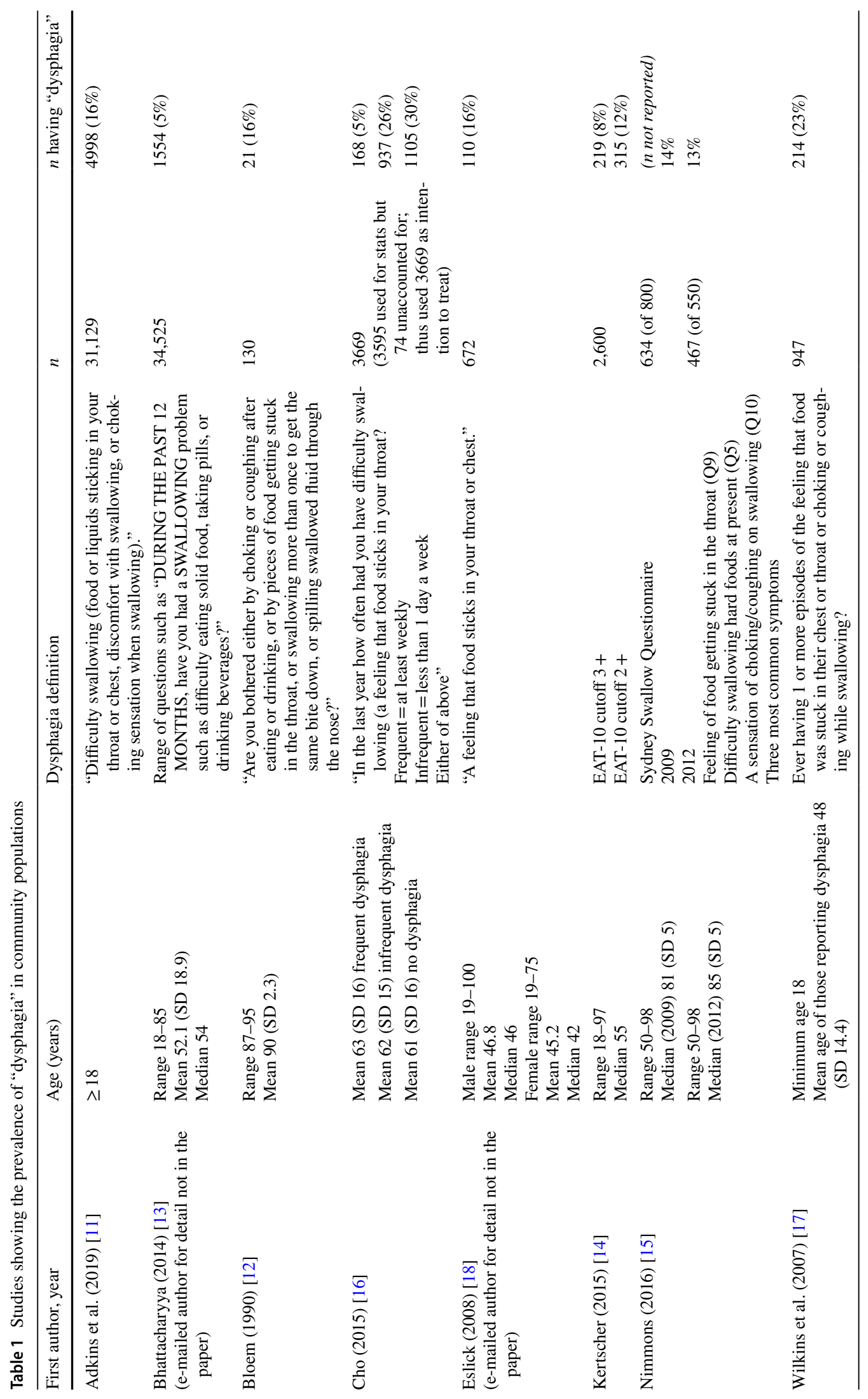




\section{Ethical Approval}

Project (PRO13050556) was reviewed by the University of Pittsburgh Institutional Review Board. Based on the information provided, this project met all the necessary criteria for an exemption and was designated as "exempt" under Section 45 CFR 46.101(b)(2) Tests, surveys, interviews, observations of public behavior on the 18th February 2014.

\section{Procedure}

The EAT-10 was converted into an online survey using Survey Monkey®. Respondents were invited to score statements on a five-point scale from "strongly disagree" to "strongly agree" and to add free comments yielding anonymous data. The survey was sent out to all the contacts known to the authors and continued onward dissemination was encouraged. The survey included demographics questions: age, sex, country of residence, nationality, medications, and previous medical history.

The presence of swallowing problems was analyzed according to Belafsky et al., with a cut-off score for "pathological" $\geq 3$ [9]. Recent work has looked at a cutoff of $\geq 2$ on the EAT-10 [14], but the literature is limited. We chose to keep our data at the $\geq 3$ cutoff to allow comparison with a more sizable body of previous sources.

\section{Data Analysis}

The data collected are categorical in nature and observational, i.e., a point in time. The data were analyzed using IBM SPSS Statistics for Windows, Version 26.0. software with the Mann-Whitney $U$ test for comparison across groups, and Spearman's rho correlation for non-parametric data [26]. We have analyzed the data using EAT-10 cutoff at $\geq 3$ indicating a swallow problem. An a priori two-tailed alpha level for significance was set at $p<0.05$.

\section{Results}

\section{Participant Characteristics and Geographical Location}

Over the period from March 2014 to October 2017, we received 2054 completed surveys (of which 32 reported ages outside of 18-65 or undeclared) resulting in a study pool of 2022 participant responses. Declared sex of the participants was 1648 reported as female (364 reported as male, 10 reported as both).

Reported scores from the EAT-10 across the whole sample of 2022 participants, and the subgroup scoring $\geq 3$ ( $n=337,17 \%$ of total sample), are shown in Table 2. Of
Table 2 EAT-10 scores for whole sample and those scoring $\geq 3$

\begin{tabular}{lll}
\hline & Sample & EAT-10 $\geq 3$ \\
\hline$N^{*}$ & 2022 & 337 \\
Median EAT-10 scores & 0 & 5 \\
Mode EAT-10 scores & 0 & 3 \\
Range EAT-10 scores & $0-36$ & $3-36$ \\
Mean (SD) EAT-10 scores & $1.57(3.49)$ & $7.02(5.91)$ \\
Age (years) & - & - \\
Median & 34 & 36 \\
Mode & 27 & 22 \\
Range & $18-65$ & $19-65$ \\
Mean (SD) & $37.12(12.40)$ & $37.81(13.21)$ \\
\hline
\end{tabular}

$* N$ for further results separated by reported sex excludes 10 participants who reported both sex options

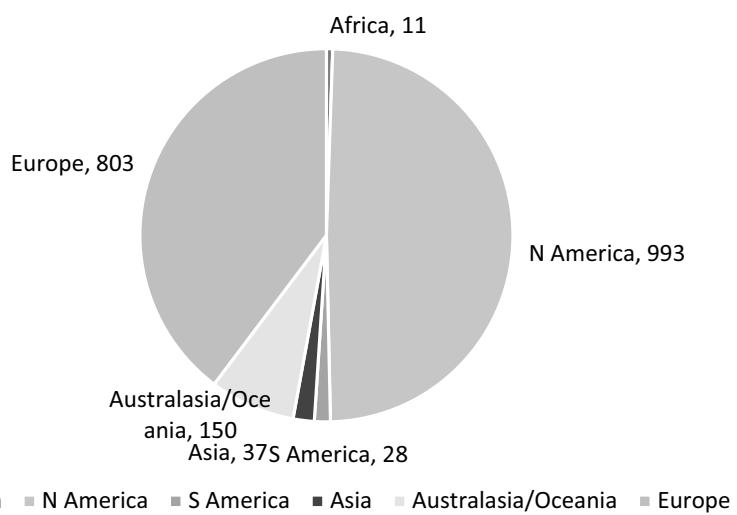

Fig. 1 Global responses by continent for all 2022 participants

these 337 responses, 166 selected n/a to the existing medical conditions where the options were head injury, stroke, Parkinson's disease, motor neuron disease, head and neck cancer, heartburn, other (please specify free text), or n/a (not applicable).

Responses were received from Africa, North America, South America, Asia, Australia/Oceania, and Europe (see Fig. 1). Responses with scores $\geq 3$ are shown in Fig. 2. As a proportion of the total responses from a continent, the "dysphagia" scores were similar with North America at $19 \%$ (191/993), Australasia/Oceania at 15\% (23/150), and Europe at 13\% (108/803). We chose not to report the proportions of the three lowest scoring continents as the numbers were so small.

For the 2012 participants who declared male or female (not both), further analysis was carried out to examine the relation between declared sex and EAT-10 scores (see Table 3). Across the group, female scores: median 0.00, mean 1.56, SD 3.338, and male scores: median 0.00, mean 1.62, SD 4.161. Using a Mann-Whitney $U$ test to compare 


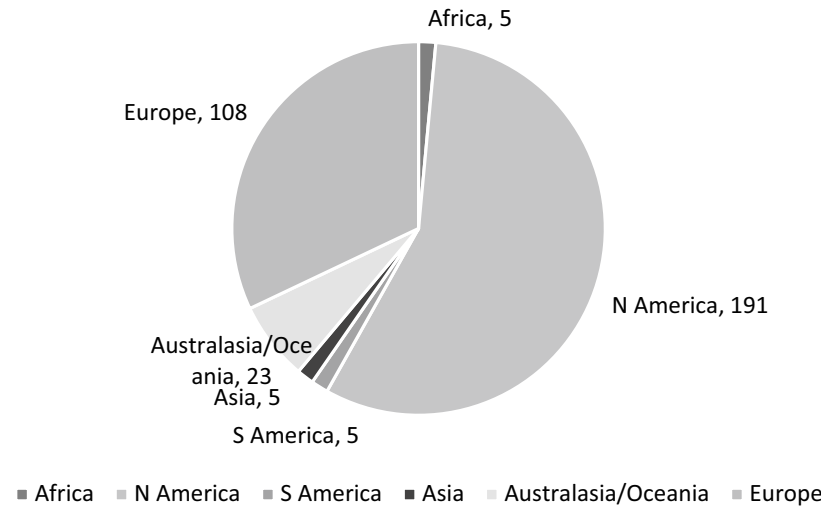

Fig. 2 EAT-10 scores $\geq 3$ by continent

Table 3 Prevalence of EAT-10 $\geq 3$ scores by declared sex

\begin{tabular}{llllll}
\hline Score & 0 & 1 & 2 & $\geq 3$ & Total \\
\hline Male $(n=364)$ & 225 & 55 & 26 & 58 & 364 \\
Female $(n=1648)$ & 865 & 318 & 187 & 278 & 1648 \\
Total & 1090 & 373 & 213 & 336 & 2012 \\
\hline
\end{tabular}

groups, the overall female score was higher than that of the overall male and was statistically significant: $U$ (Nfemale $=1648$, Nmale $=364)=275,420.000, z=-2.677$, $p=0.007$. Declared sex was not statistically significantly associated with non-pathological vs. pathological EAT-10 score: $U($ Nfemale $=1648$, Nmale $=364)=297,132.000$, $z=-0.433, p=0.665$.

Spearman's rho correlation coefficient was used to assess the relationship between age and EAT-10 score. In females, there was no significant correlation between the two $r_{\mathrm{s}}=-0.043, p=0.079, N=1648$. In males, there was no significant correlation between the two $r_{\mathrm{s}}=-0.003, p=0.952$, $N=364$.

We split our data across decades: 18-30 years, $31-40$ years, $41-50$ years, and $51-65$ years. There is no evidence that there is a physiological difference across these age bands; this was purely to allow comparison with the previous work. Mann-Whitney $U$ test was used to examine the relation between declared sex and EAT-10 scores in these age bands and no statistically significant differences were found (see Table 4).

\section{Participant Comments from Free Text}

Several participants commented that they should have been asked if they had a swallow problem at the start of the survey as then they would not have completed it. Interestingly several respondents scored below the cutoff but expressed considerable concern about their swallow as a whole, and/ or with features not captured in the survey. The degree to which a person was concerned about their swallow was not necessarily reflected in their EAT-10 score.

Comments from those with a score of 2 or less showed a range of views from no concern, to understanding of why there might be an issue, to great concern:

I now cough (occasionally choke) 3-4 times per day; usually thin liquids. This is a change from my 50s! (no diagnosis, $E A T-10=2$ )

Occasionally certain textures e.g. small particules [sic] of apple or rice will enter back of nasal cavity if rushing, not paying attention and not chewing properly. Uncomfortable sensation but does not put me off eating these consistencies. Very fast eater-sometimes have regurgitation immediately post meals. (no diagnosis, EAT-10=1).

I'll go through periods of choking on things- eg several times a week? due to distractibility or hypersensitivity from the original bout of choking. Otherwise the norm would be choking maybe a few times a year. (no diagnosis, EAT-10=1).

I have noticed more often in about the past 6 months that I tend to "spontaneously" aspirate saliva (even just when sitting... all of a sudden i'll feel some sneak in the airway and have a coughing jag) (no diagnosis, EAT-10=1).

Of 337 responses with a score or $\geq 3$, there were 166 with no reported illnesses of whom 16 participants reported that they did not have a swallow problem in the comments box and shared other information which was categorized into fear $(n=3)$, embarrassment $(n=2)$, eating
Table 4 Age (split into age bands) of those scoring $\geq 3$ on EAT-10 by declared sex

\begin{tabular}{lllll}
\hline Age (years)/EAT-10 by sex & $18-30$ & $31-40$ & $41-50$ & $51-65$ \\
\hline Male $<3$ & 89 & 75 & 62 & 80 \\
Female $<3$ & 554 & 334 & 248 & 234 \\
Male $\geq 3$ & 13 & 18 & 7 & 20 \\
Female $\geq 3$ & 114 & 57 & 44 & 63 \\
Total & 770 & 484 & 361 & 397 \\
Mann-Whitney & $39,358.500$ & $14,469.000$ & 7409.000 & $12,851.000$ \\
$U$ statistic & & & & \\
$z$ value & -1.094 & -1.143 & -1.055 & -0.257 \\
$p$ value & 0.274 & 0.253 & 0.292 & 0.797 \\
\hline
\end{tabular}


in public but not mentioning fear/embarrassment $(n=3)$, producing froth, sputum etc. $(n=1)$, were on a feeding tube/TPN $(n=2)$, concern/anxiety $(n=1)$, and coughing who scored 0 on the EAT-10 cough question (4). Comments included:

Sometimes I will choke on things like onion or lettuce. I'm always the last person to finish eating as I chew a lot to minimize chances of choking or food getting stuck. (no diagnosis EAT-10=12).

I take ages to eat a main course (30-40 min)—everyone else has finished and I am aware that I am only half way through and I am unable to eat any faster. This is embarrassing and I often leave food even though I am still hungry. I ask for child portions and look for items on the menu that are easy to eat. (no diagnosis EAT-10=17).

I believe Achalasia is more common than prevalence figures quoted in mainstream literature. My condition was masked by pregnancy and eventually severe weight loss led to premature birth. (late diagnosis EAT-10=22).

\section{Discussion}

This is the largest study to date, covering the widest geographical range, of self-reported swallowing difficulties in community populations. The proportion of people in North America (19\%), Australasia/Oceania (15\%), and Europe (13\%) who reported an EAT-10 score high enough to be classed as pathological is in line with the previous studies (see Table 1).

We did not set out to address the health care seeking behaviors of participants but did receive reports that people were embarrassed to approach health care providers. We know from previous research that a large proportion of people typically do not seek help despite a experiencing a significant impact on physical and psychological well-being. Wilkins et al. found $2 \%$ of 947 people $\geq 18$ years of age reported dysphagia at least several times a month and $46 \%$ had not reported the problem to their general practitioner [17]. Adkins et al. reported 2445 of 4998 (49\%) survey participants had not consulted a health care practitioner, and of those who did 2449 (96\%) had health insurance [11]. In societies where health care is a business, those with financial means (independent wealth or insurance) are more likely to see help earlier and continue to access health care support. This has direct consequences for the impact and treatment of the disease underlying dysphagia.

The degree to which a person was concerned about their swallow was not necessarily reflected in their EAT-10 score. Of interest are the participants who:
1. scored below the cutoff but expressed considerable concern about their swallow, and/or with features not captured in the survey;

2. scored above the cutoff and reported no disease; and

3. scored above the cutoff, reported no disease, and stated in the free text that they had no swallow problems.

Group 1 may have clinical issues and the survey tool was not capturing them, or they may not have organic disease but still have concerns. These contrast with the people in Group 2: what is causing their higher scores? This may relate to features of the EAT-10 questions that people report but do not perceive as problematic. It may be due to swallow issues being lower on a list of issues that a person is dealing with.

Group 3 are possibly the most interesting. How can you get such high scores and state that you have no swallow problem? This is not uncommon in clinical practice where you have patients who tell you they have no swallow problem but on more subtle questioning they share that they now avoid certain food types, have changed their posture, always take drinks to help move material down their throats, etc. And you may say again "so do you have any problems swallowing?" to which they still say no. Our study focused on the age range 18-65 years but similar to many other areas of health people accommodate to changes as they get older presuming them to be a part of the aging process [27]. In common with many other systems in the body, we do not normally see a simple decrease in ability with age and so changes should be investigated.

All three of these groups perhaps represent the disconnect between how professionals view the swallow process and how patients experience and think about it. This mirrors the situation in the world of voice disorders where clinicians and patients disagree, and instrumental and subjective measures do not align [28]. How can this be so we might ask? One aspect is that the two realities (patient experienced and clinician observed) are different, so tools are not measuring the same things. The other aspect is that definitions are so unclear. This is the case in the world of swallowing disorders even among professionals. It is no wonder that patients do not report things that fit our screening and assessment tools particularly when we are looking at complex and subtle systems.

There are people in the community with swallow difficulties who are not receiving support. Dysphagia professionals are aware that the disorder exists where there is an underlying condition. The underlying condition may be insidious and not yet detected, or may be stark such as a stroke but the professionals involved have missed the minor swallow issues in the midst of more weighty problems. There are people who have a condition who do not seek help. This is of particular concern in the current climate where people are avoiding going to hospital for fear of catching COVID-19 
or overburdening already stretched services [29]. A recent study attempted to track patient reported swallow difficulties and general health-related quality of life issues across six months [30]. The authors acknowledged limitations in the work including aspects of the EAT-10 in detecting change in people with mild dysphagia for example, but it was an important step in addressing symptoms from the perspective of the patient rather than the clinical or research professional.

One previous study found a peak in reported dysphagia in the 40-49 year old age band [18]. We did not find evidence of any differences across age bands. This previous result may be an artifact of the questionnaire used: comparison of true numbers but what does true mean? The variation in definitions used by authors, shared with participants and others, limits cross study comparison and possibly creates confusion.

\section{Limitations}

Study limitations include use of the EAT-10 itself. At the time the work was being designed we needed a simple tool, suitable for online survey work to reach as large a spread as possible. Recent work has shown that the psychometric properties of the EAT-10 are poor [31-34], thus going forward investigators should consider alternatives. Investigators also need to carefully consider what their definition of dysphagia is, because how this is worded will control the findings.

Further limitations include the inherent volunteer bias that surveys are subject to. The large number of people with low scores shows that the survey was not just taken by those with a vested interest, i.e., those with the condition. The survey is likely to have captured a narrow slice of society: those with access to the internet, and through connections to existing participants.

Respondents to the survey were largely from the USA, Europe, and Australasia/Oceania. Access to, participation in, and perceptions of swallowing difficulties are likely to vary across countries and cultures [35]. Unlike say a broken leg, the swallow process and its relation to the acts of eating and drinking are much less concrete. A person's perception of their swallow and whether it is problematic is influenced by more than biomechanics. Knowledge of what support is available, how health care professionals tend to react, how issues might influence employment etc., all influence the lens through which a person might engage with services [36].

\section{Clinical Significance}

We need to be careful to not medicalize the ends of the range of normality in the swallow: difference is not necessarily a disorder. Nevertheless, there were a number of respondents who shared the significant impact on their lives. We may need a two pronged approach: partly to raise awareness with the public that swallow difficulties should be checked out, without causing undue concern. Secondarily to educate professionals to ask about how people are managing with eating and drinking. This is difficult in the absence of obvious disease. We in the dysphagia community understand the subtleties and relationship of aspects of the swallow to other areas of health and illness, but not everyone does. Education of the broader health care community is an ongoing process, although promise is being shown by early work such as the 4QT [37]. This tool is designed to be quick and used by any member of a health care team. The 4QT is still in the pilot stage with good sensitivity but poor specificity, which in a screen designed to detect issues for onward assessment is not a bad thing.

We have a current and real concern: rehabilitation in major pandemics where the respiratory system is affected, e.g., COVID-19. The swallow process is inherently interconnected with the respiration systems [38]. Mechanical damage due to intubation or issues of respiratory-swallow coordination will need addressing in long-term rehabilitation efforts [39]. People with COVID-19 related and unrelated dysphagia will need care [40]. There is emerging evidence on the pre-COVID-19 conditions that lead to poorer outcomes with an existing baseline of neurological conditions [41] particularly those with pre-existing cerebrovascular disease [42] both of which we know to be high-risk areas for swallow impairments.

\section{Future Directions}

There is enough published literature to consider a systematic review of all the studies focusing on people living in the community. This may be confounded by the range of definitions of what "dysphagia" is. A clear definition of dysphagia or clearly delineated subtypes is required. This would support future researchers to make the many subtly different studies comparable. This would be a worthy aim for the national and international dysphagia research forums. Such a structure would also support the development or modification of existing screening and assessment tools, again to allow for easier comparison.

A formal comparison of clinically assessed and/or instrumentally measured features of the swallow in comparison to what people report with—and without—swallow impairments would contribute to the teasing out of what is a swallow problem. In whose eyes does the dysphagia lie: the patient or the professional? Addressing the issue of perception has parallels to, and would contribute to, other areas of health care. These are far more complex issues than 
the biomechanics of which we know so much. Gaining clarity here might contribute back to definitions and thus guide research to answer the most impactful questions regarding the care of people with swallow difficulties.

Acknowledgements The authors thank all those who took the time to complete the survey and share it with other participants. They would also like to thank the University of Pittsburgh Communication Science and Disorders students Katy Conlon and Danielle Johnson who assisted in data management, and the Occupational Therapy class at the University of Pittsburgh who piloted the online survey for us.

Open Access This article is licensed under a Creative Commons Attribution 4.0 International License, which permits use, sharing, adaptation, distribution and reproduction in any medium or format, as long as you give appropriate credit to the original author(s) and the source, provide a link to the Creative Commons licence, and indicate if changes were made. The images or other third party material in this article are included in the article's Creative Commons licence, unless indicated otherwise in a credit line to the material. If material is not included in the article's Creative Commons licence and your intended use is not permitted by statutory regulation or exceeds the permitted use, you will need to obtain permission directly from the copyright holder. To view a copy of this licence, visit http://creativecommons.org/licenses/by/4.0/.

\section{References}

1. Fiese BH, Foley KP, Spagnola M. Routine and ritual elements in family mealtimes: contexts for child well-being and family identity. New Dir Child Adolesc Dev. 2006;111:67-89.

2. Wilk RR. "Real Belizean food": building local identity in the transnational caribbean. Am Anthropol. 1999;101(2):244-55.

3. Daniels SK, et al. Mechanism of sequential swallowing during straw drinking in healthy young and older adults. J Speech Lang Hear Res. 2004;47(1):33-45.

4. Dodds WJ, Stewart ET, Logemann JA. Physiology and radiology of the normal oral and pharyngeal phases of swallowing. Am J Roentgenol. 1990;154(5):953-63.

5. Martin BJW, et al. Coordination between respiration and swallowing: respiratory phase relationships and temporal integration. J Appl Physiol. 1994;76(2):714-23.

6. Roden DF, Altman KW. Causes of dysphagia among different age groups: a systematic review of the literature. Otolaryngol Clin N Am. 2013;46(6):965-687.

7. Kalf JG, et al. Prevalence of oropharyngeal dysphagia in Parkinson's disease: a meta-analysis. Parkinsonism Relat Disord. 2012;18(4):311-5.

8. Takizawa C, et al. A systematic review of the prevalence of oropharyngeal dysphagia in stroke, Parkinson's disease, Alzheimer's disease, head injury, and pneumonia. Dysphagia. 2016;31(3):434-41.

9. Belafsky PC, et al. Validity and reliability of the eating assessment tool (EAT-10). Ann Otol Rhinol Laryngol. 2008;117(12):919-24.

10. Wallace KL, Middleton S, Cook IJ. Development and validation of a self-report symptom inventory to assess the severity of oralpharyngeal dysphagia. Gastroenterology. 2000;118(4):678-87.

11. Adkins C, et al. Prevalence and characteristics of dysphagia based on a population-based survey. Clin Gastroenterol Hepatol. 2020;18(9):1970-9.

12. Bloem BR, et al. Prevalence of subjective dysphagia in community residents aged over 87. BMJ. 1990;300(6726):721-2.
13. Bhattacharyya $\mathrm{N}$. The prevalence of dysphagia among adults in the United States. Otolaryngol Head Neck Surg. 2014;151(5):765-9.

14. Kertscher B, et al. Prevalence of oropharyngeal dysphagia in the Netherlands: a telephone survey. Dysphagia. 2015;30(2):114-20.

15. Nimmons D, et al. A longitudinal study of symptoms of oropharyngeal dysphagia in an elderly community-dwelling population. Dysphagia. 2016;31(4):560-6.

16. Cho SY, et al. Prevalence and risk factors for dysphagia: a USA community study. Neurogastroenterol Motil. 2015;27(2):212-9.

17. Wilkins $\mathrm{T}$, et al. The prevalence of dysphagia in primary care patients: a HamesNet Research Network study. J Am Board Fam Med. 2007;20(2):144-50.

18. Eslick GD, Talley NJ. Dysphagia: epidemiology, risk factors and impact on quality of life: a population-based study. Aliment Pharmacol Ther. 2008;27(10):971-9.

19. Beltran-Corbellini A, et al. Acute-onset smell and taste disorders in the context of COVID-19: a pilot multicentre polymerase chain reaction based case-control study. Eur J Neurol. 2020. https://doi.org/10.1111/ene.14273.

20. Kiekens C, et al. Rehabilitation and respiratory management in the acute and early post-acute phase. "Instant paper from the field" on rehabilitation answers to the Covid-19 emergency. Eur J Phys Rehabil Med. 2020;56:323.

21. Lechien JR, et al. Olfactory and gustatory dysfunctions as a clinical presentation of mild-to-moderate forms of the coronavirus disease (COVID-19): a multicenter European study. Eur Arch Otorhinolaryngol. 2020;277:2251.

22. Luers JC, et al. Olfactory and gustatory dysfunction in coronavirus disease 19 (COVID-19). Clin Infect Dis. 2020. https://doi. org/10.1093/cid/ciaa525.

23. Qiu C, et al. Olfactory and gustatory dysfunction as an early identifier of COVID-19 in adults and children: an international multicenter study. Otolaryngol Head Neck Surg. 2020. https:// doi.org/10.1177/0194599820934376.

24. Speth MM, et al. Olfactory dysfunction and sinonasal symptomatology in COVID-19: prevalence, severity, timing, and associated characteristics. Otolaryngol Head Neck Surg. 2020;163(1):114-20.

25. Dietsch A, et al. Taste manipulation and swallowing mechanics in trauma-related sensory-based dysphagia. J Speech Lang Hear Res. 2019;62(8):2703-12.

26. IBM Corp, IBM SPSS Statistics for Windows, V26.0. 2019, IBM Corp: Armonk, NY.

27. Chen PH, et al. Prevalence of perceived dysphagia and quality-of-life impairment in a geriatric population. Dysphagia. 2009;24(1):1-6.

28. Karnell MP, et al. Reliability of clinician-based (GRBAS and CAPE-V) and patient-based (V-RQOL and IPVI) documentation of voice disorders. J Voice. 2007;21(5):576-90.

29. Hautz WE, et al. Barriers to seeking emergency care during the COVID-19 pandemic may lead to higher morbidity and mortality: a retrospective study from a Swiss university hospital. Swiss Med Wkly. 2020;150:w20331.

30. Bartlett RS, Moore JE, Thibeault SL. Temporal analysis of factors associated with EAT-10 in outpatients with oropharyngeal dysphagia from a tertiary care clinic. Dysphagia. 2018;33(4):457-67.

31. Cordier R, et al. Evaluating the psychometric properties of the eating assessment tool (EAT-10) using Rasch analysis. Dysphagia. 2017;32(2):250-60.

32. Kean J, et al. An introduction to Item Response Theory and Rasch Analysis of the Eating Assessment Tool (EAT-10). Brain Impair. 2018;19:91-102.

33. Wilmskoetter J, et al. Construct validity of the Eating Assessment Tool (EAT-10). Disabil Rehabil. 2019;41(5):549-59. 
34. Hansen T, Kjaersgaard A. Item analysis of the Eating Assessment Tool (EAT-10) by the Rasch model: a secondary analysis of crosssectional survey data obtained among community-dwelling elders. Health Qual Life Outcomes. 2020;18(1):139.

35. Hofstede GH. Culture's consequences: comparing values, behaviors, institutions, and organizations across nations. 2nd ed. London: SAGE; 2001.

36. Touboul-Lundgren $P$, et al. Identification of cultural determinants of antibiotic use cited in primary care in Europe: a mixed research synthesis study of integrated design "Culture is all around us." BMC Public Health. 2015; 15:908.

37. Tsang K, et al. A new simple screening tool-4QT: can it identify those with swallowing problems? A pilot study. Geriatrics. 2020;5:11.

38. Martin-Harris B, et al. Breathing and swallowing dynamics across the adult lifespan. Arch Otolaryngol. 2005;131(9):762-70.

39. Ceravolo MG, et al. Rehabilitation and Covid-19: the Cochrane Rehabilitation 2020 rapid living systematic review. Eur J Phys Rehabil Med. 2020. https://doi.org/10.23736/S1973 $-9087.20 .06501-6$.
40. Fritz MA, et al. Moving forward with dysphagia care: implementing strategies during the COVID-19 pandemic and beyond. Dysphagia. 2020. https://doi.org/10.1007/s00455-020-10144-9.

41. Butala N. Neurological aspects of Coronavirus Infectious Disease 2019 (COVID-19). Innov Clin Neurosci. 2020;17(4-6):13-5.

42. Patel U, et al. Pre-existing cerebrovascular disease and poor outcomes of COVID-19 hospitalized patients: a meta-analysis. J Neurol. 2020. https://doi.org/10.1007/s00415-020-10141-w.

Publisher's Note Springer Nature remains neutral with regard to jurisdictional claims in published maps and institutional affiliations.

Paula Leslie PhD, MA (Bioethics) FRCSLT, CCC-SLP

David G. Smithard BSc, MB, MD, FRCP, FRCSLT(Hon) 